\title{
D-07
}

\section{ANÁLISIS DE LOS BENEFICIOS ECONÓMICOS DE UN SERVICIO DE ASESORAMIENTO AL REGANTE (SAR)}

\author{
Silva do Nascimento, A. K. ${ }^{1}$ Tarjuelo Martín-Benito, J. M. $^{2}$ Ribeiro Vieira Lima, S. C. ${ }^{3}$ \\ Martinez-Romero, $A^{4}{ }^{4}$
}

${ }^{1}$ Estudiante de Doctorado, Universidad Castilla-la Mancha, anakelliane02@gmail.com

${ }^{2}$ Dr. Ingeniero Agrónomo, Catedrático de Universidad; Departamento de Producción Vegetal y Tecnología Agraria, UCLM; jose.tarjuelo@uclm.es

${ }^{3}$ Investigador, Instituto de Pesquisa e Inovação na Agricultura Irrigada (INOVAGRI), silviocarlos@inovagri.org.br

${ }^{4}$ Dr. Ingeniero Agrónomo, Profesor Asociado; ElIAB, Departamento de Mecánica Aplicada e Ingeniera de Proyectos, UCLM; angel.mromero@uclm.es

\section{Resumen}

Un Servicio de Asesoramiento al regante (SAR) tiene como objetivo principal la optimización del uso del agua en la agricultura, dando respuesta a las demandas tecnológicas de los regantes. El objetivo de este trabajo es analizar y cuantificar, para una parcela tipo de cebolla, los beneficios económicos de un Servicio de Asesoramiento al Regante (SAR), a través de un análisis comparativo de distintos manejos del riego en parcela. En el año 2015, en una parcela de cebolla ubicada en una Comunidad de Regantes (CCRR) de la provincia de Albacete se realizó el seguimiento del desarrollo fenológico del cultivo, y de la humedad en el suelo, que junto con los datos edáficos y climáticos permitió planificar la programación de riegos. Se plantearon cuatro escenarios comparativos: E1 (datos reales de manejo de riego en parcela), E2 (simulación tomando como referencia las recomendaciones semanales del SAR de Castilla-La Mancha (SIAR $\mathrm{CM}_{\mathrm{CM}}$ ), E3 (simulación con igual aporte que en el E1, variando la frecuencia y la dosis de riego) y E4 (simulación de riego optimizado realizando un Balance de agua en el suelo con el módulo de programación de riegos del programa MOPECO). Los costes de producción del cultivo se calcularon a partir de los datos facilitados por el agricultor, permitiendo interpretar indicadores tales como: el Suministro Relativo de Agua, Índice de Producción, Margen Bruto y la Productividad Agronómica del Agua. Los resultados indican que frente a un manejo sin seguir las recomendaciones de un SAR, los datos facilitados por el SIAR $_{\mathrm{CM}}$ y una correcta programación pueden influir en un aumento de hasta $24 \%$ del rendimiento, una mayor Productividad Agronómica del Agua $\left(8,45 \mathrm{~kg} / \mathrm{m}^{3}\right.$ respecto a real obtenida de $7,14 \mathrm{~kg} / \mathrm{m}^{3}$ ) y un incremento del Margen Bruto de hasta un $50 \%$.

\section{1) Introducción}

El uso eficiente de agua y energía en la agricultura está ganando importancia debido a una tendencia limitante de la disponibilidad de agua para usos agrícolas, así como el aumento de los costes de energía. Estos aspectos condicionan la viabilidad de las actividades de riego en muchas zonas del mundo, entre ellas la región de Castilla-La Mancha (C-LM) (Ortega et al., 2004; Martínez-Valderrama et al., 2011).

A partir de la necesidad de una gestión adecuada de los cultivos que permite obtener producciones adecuadas y hacer un uso racional y eficiente de los recursos hídricos, los SAR se presentan como una herramienta adecuada para la optimización del uso del agua ofreciendo información para establecer una correcta programación de riegos.

Los SARs ofrecen apoyo técnico a los agricultores con el objetivo de conseguir un uso eficiente y racional de los medios de producción, especialmente de los fertilizantes, del agua, y 
de la energía, aumentando además la rentabilidad económica del regadío. Realizan una labor de transferencia tanto de información técnica, como de nuevas tecnologías que pueden aplicarse directamente en sus explotaciones, ayudando a optimizar su manejo, lo que repercute en beneficios de índole económicos y medioambientales.

La gestión óptima del riego en los cultivos implica la integración de diversos factores relacionados con la producción y calidad, que conducen al óptimo económico. Por ello es necesario definir y conocer las necesidades de agua de los cultivos, la interacción sueloplanta-atmósfera, efecto del déficit hídrico en la producción y calidad o el sistema de aplicación del agua de riego. El aumento de los costes de producción, especialmente los de la energía eléctrica asociada a los sistemas de riego presurizados, así como la limitación de las dotaciones de agua para el riego, está condicionando la implantación de cultivos y el manejo del riego en muchas zonas para hacer un uso más eficiente del agua. Uno de los principales problemas para que los SARs alcancen los objetivos potenciales, es conseguir que la información generada llegue a los usuarios a los que está destinada, así como que ésta, sea correctamente interpretada y aplicada para conseguir los objetivos perseguidos.

En general se asume la rentabilidad de los SARs, pero los trabajos que cuantifican los beneficios económicos de los mismos son limitados. El objetivo de este trabajo, que se engloba dentro de otro más amplio, es cuantificar y valorar los beneficios económicos de un SAR tomando como ejemplo un cultivo de cebolla, realizando un análisis comparativo de distintos manejos del riego en parcelas realizados por agricultores, comparándolo con un manejo en el que se tengan presentes las recomendaciones semanales de un SAR, y otro en el que se realice una programación de riegos más tecnificada, basada en un balance simplificado de agua en el suelo. Para ello se utilizan modelos de simulación de cultivo previamente calibrados experimentalmente tales como el modelo MOPECO (Domínguez et al., 2012).

\section{2) Materiales y métodos}

\section{1) Zona de Estudio}

Para la realización del estudio se realizó el seguimiento fenológico y manejo del riego en una parcela de cebolla de 2,48 ha durante la campaña de riegos de 2015 , regada con cobertura total enterrada en marco $16,50 \mathrm{~m} \times 17,40 \mathrm{~m}$, dentro de una red colectiva de riego a la demanda ubicadas en el término municipal de Tarazona de la Mancha (Albacete) (Figura 1).

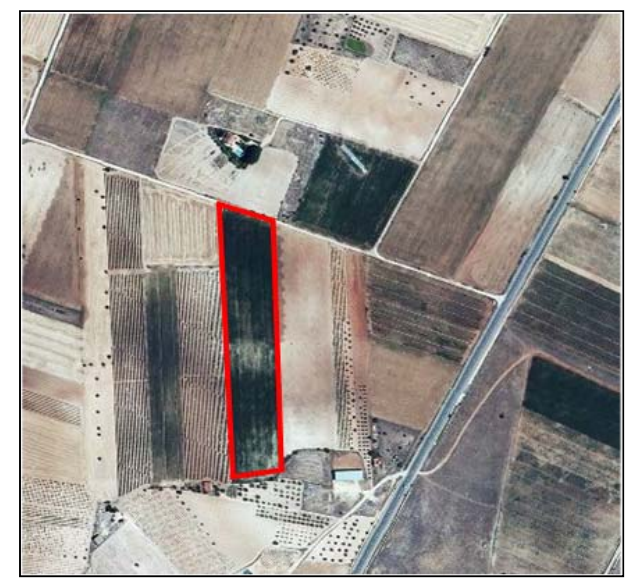

Figura 1. Parcela de seguimiento cultivada con cebolla; (Fuente: SigPac (http://sigpac.mapa.es/fega/visor/).

\section{2) Programación de Riego}

La programación de riegos se estableció siguiendo la metodología FAO del balance simplificado de agua en el suelo, contrastado con la medición de los valores del potencial 
hídrico en el suelo con sondas Watermark, estimando la equivalencia del contenido volumétrico de agua en el suelo en base a los valores medidos de densidad aparente del suelo y las curvas características de humedad con cámara de presión.

La evapotranspiración de referencia se estimó a partir de los datos registrados en la estación meteorológica de La Gineta integrada en la red del Servicio de Información Agroclimática para el Regadío por el método Penman Monteith (Allen et al., 1998). El balance hídrico se calculó mediante el módulo de programación de riegos del programa MOPECO que requiere datos sobre el suelo, clima y el cultivo. Los valores de Kc fueron obtenidos en base a las recomendaciones de FAO (Allen et al., 1998), adaptadas a los resultados de seguimiento realizados por el SIAR $R_{C M}$, para tener en cuenta las condiciones locales (Domínguez et al. 2012). Los valores de Kc utilizados en el cultivo fueron: en la etapa de establecimiento 0,65; en la Etapa de crecimiento vegetativo 0,65-1,20: en la etapa media 1,20 y en la etapa final $1,20-0,75$.

En la parcela del estudio se instaló, en un punto representativo de las condiciones medias de funcionamiento del sistema de riego, un transductor de presión para poder conocer el tiempo de riego, las condiciones de presión, y determinar la cantidad de agua aplicada. También se realizó al inicio de la campaña una evaluación en el sistema de riego.

Para analizar el posible efecto de distintos manejos, fueron considerados escenarios comparativos:

a) Escenario 1 (E1): este escenario presenta el riego real aplicado por el agricultor, basado en la experiencia.

b) Escenario 2 (E2): Simulación tomando como referencia las recomendaciones generales de consumos netos semanales ofrecidas por el SIAR $\mathrm{CM}_{\mathrm{M}}$. Los consumos publicados se refieren a valores de la semana anterior. Se ha establecido una frecuencia máxima de aplicación de 5 días (un riego a la semana) y mínima de 2 días (3 o 4 riegos por semana). El tiempo de riego utilizado varía entre $1 \mathrm{~h}(6 \mathrm{~mm})$ y $4 \mathrm{~h}(24 \mathrm{~mm})$.

c) Escenario 3 (E3): Simulación a partir del volumen real aplicado por el agricultor (E1), pero con intervalos entre riegos y dosis de riego distintos de los aplicados. El tiempo de riego para este manejo se ha establecido entre $1 \mathrm{~h}(6 \mathrm{~mm})$ y $3 \mathrm{~h}(18 \mathrm{~mm})$, con un intervalo entre riegos de entre 2 días y 4 días.

d) Escenario 4 (E4): Simulación aplicando un riego optimizado para la máxima producción, a partir de un balance de agua en el suelo, con ayuda del modelo MOPECO. El tiempo de riego para este manejo se ha establecido entre $1 \mathrm{~h}(6 \mathrm{~mm})$ y $5 \mathrm{~h}(30 \mathrm{~mm})$, con un intervalo entre riegos entre 1 días y 4 días.

MOPECO utiliza el modelo propuesto por Stewart et al. (1977) que estima el rendimiento del cultivo en función de la relación entre evapotranspiración real y la evapotranspiración máxima (ETa/ETm) en las diferentes etapas de crecimiento del cultivo. Cuando $\mathrm{ETa}<\mathrm{ETm}$ la planta sufre estrés hídrico que puede provocar un descenso en el rendimiento final (rendimiento real $(\mathrm{Ya})<$ rendimiento potencial $(\mathrm{Ym})$. El rendimiento potencial depende de la zona del estudio (Domínguez et al., 2012).

\section{3) Evaluación de los costes}

Los costes del SAR son muy distintos dependiendo de diversos factores, tales como los objetivos planteados, el nivel de información generada o la estructura y disposición de las explotaciones. En este trabajo los costes de producción del cultivo, para los distintos escenarios, se calcularon utilizando las informaciones facilitadas por los productores sobre la materia prima, abonos, labores y aplicación del agua. La metodología utilizada para calcular los costes fue la facilitada por de Juan et al. (2003). Los costes fueron clasificados en Costes externos, que se refieren a los costes como semillas, fertilizantes, fitosanitarios y agua; Costes calculados, que son los de mano de obra y maquinaria; Costes estimados, que son los seguros agrícolas y alquiler de maquinarias y Costes fijos, que hace referencia a una tasa fija por tratarse de una Comunidad de Regantes. La cuantificación de los ingresos fueron obtenidos a través de la multiplicación entre la producción y el precio de venta, que en este trabajo fue utilizado la cotización informada por el agricultor. Se agregó al resultado 
de los ingresos el valor de la PAC, que es una ayuda proporcionada por la Unión Europea a los agricultores. Toda esta información se analizó utilizando distintos indicadores para evaluar la repercusión económica de una correcta programación de riegos. Los indicadores planteados fueron:

e) Suministro Relativo de Agua (SRA): muestra la relación entre el agua que entra al sistema (precipitación y riego) y el agua requerida (evapotranspiración), indicando la relación entre la cantidad de agua disponible o utilizada y la cantidad de agua necesaria para la producción (Levine, 1982). Este indicador fue calculado por la Ecuación 1.

$$
S R A=(R G+P e) E T c^{-1}
$$

Donde SRA: suministro relativo de agua (\%); RG: agua de riego ( $\left.\mathrm{m}^{3} / \mathrm{ha}\right)$; Pe: precipitación efectiva $\left(\mathrm{m}^{3} / \mathrm{ha}\right)$. ETc: evapotranspiración de cultivo $\left(\mathrm{m}^{3} / \mathrm{ha}\right)$. La Precipitación efectiva $(\mathrm{Pe})$ se estima a través del USDA "metodología de la curva número 2" (SCS, 1972; NRCS, 2004).

La caracterización del riego aportado en función de si se ven satisfechas las necesidades de los cultivos, atiende al criterio presentado en la tabla 1:

Tabla 1. Criterio utilizado para clasificación de los escenarios según SRA

\begin{tabular}{|l|l|}
\hline Si SRA $(\%)>115 \%$ & Riego excesivo \\
\hline Si $115 \geq$ SRA $(\%) \geq 85 \%$ & Bien regado \\
\hline Si SRA $(\%)<85 \%$ & Riego insuficiente \\
\hline
\end{tabular}

f) Índice de Producción (IP), definido por Bos et. al (1994), relaciona la producción real (Cr) en $\mathrm{kg} / \mathrm{ha}$ con la producción máxima alcanzable por el cultivo $(\mathrm{Cp})$ en $\mathrm{kg} / \mathrm{ha}$. Los datos reales fueron facilitados por el agricultor y el rendimiento potencial, estimado en el modelo MOPECO. El indicador fue calculado por la Ecuación 2.

$$
\mathrm{IP}=\mathrm{Cr} / \mathrm{Cp}
$$

g) Margen Bruto de la producción (MB), El análisis de este indicador puede ser usado para comparar la utilidad de emplear diferentes tecnologías; en este caso, la tecnología ofrecida por el SIAR y la utilizada por el agricultor. El margen bruto (MB, en $€ /$ ha) fue calculado por la Ecuación 3, donde VP es el valor total de producción, incluyendo la Subvención ( $€ /$ ha) y GG son los gastos globales ( $€ /$ ha).

$$
M B=V P-G G
$$

h) Productividad Agronómica del Agua (PAA), que es claramente dependiente del cultivo, de las condiciones climáticas, y del manejo del riego llevado a cabo por el agricultor. Puede medirse mediante la relación entre la unidad de resultado conseguido y la unidad de insumo. En este caso el término productividad del agua (PA) es usado para poner de manifiesto la relación entre la cantidad del producto obtenido sobre el volumen o valor del agua aplicada (FAO, 2003). La PAA se obtuvo por la Ecuación 4.

$\mathrm{PAA}=\mathrm{RC} / \mathrm{Vr}$

En que PAA: Productividad Agronómica del Agua en $\mathrm{kg} / \mathrm{m}^{3}$; RC: rendimiento real del cultivo, en $\mathrm{kg} / \mathrm{ha}$; $\mathrm{Vt}=$ volumen de riego aplicado en $\mathrm{m}^{3} / \mathrm{ha}$, obtenido con base en la evaluación en campo.

\section{3) Resultados y discusión}




\section{1) Balance de agua en el suelo. Relaciones ETa/ETm en el cultivo}

Con respecto al E1 (manejo real en parcela), en la Figura 2 se muestra el gráfico de la evolución del contenido de agua en el suelo generado por el modelo MOPECO según la programación de riego seguida por el agricultor y las lluvias registradas en la parcela de estudio. Para facilitar la comprensión del gráfico cabe indicar que, la línea AW representa la evolución diaria de la cantidad de agua que hay disponible en el suelo para el cultivo durante su ciclo de crecimiento. Su magnitud depende de las características físicas y profundidad del suelo y la profundidad radicular alcanzada por el cultivo en cada etapa. La línea 1-p representa el nivel de agotamiento permisible de agua en el suelo. Representa el contenido de agua mínima en el suelo por encima del cual se evita el estrés hídrico en el cultivo que puede repercutir en el rendimiento del cultivo. Este valor depende del tipo de cultivo, su estado de desarrollo y de las condiciones climáticas (tomando como referencia la evapotranspiración de referencia, ETo). La línea ETa/ETm acumulada por etapa representa la relación entre evapotranspiración real del cultivo (ETa) y la evapotranspiración de un cultivo específico en condiciones de densidad y fertilización de suelo óptimas, es decir el máximo que podría evapotranspirar (Evapotranspiración máxima, ETm) en cada etapa. La línea $\mathrm{ETa} / \mathrm{ETm}$ diario representa la evolución de la relación diaria $\mathrm{ETa} / \mathrm{ETm}$; cuando este valor es inferior a 1 indica que el cultivo está sometido a estrés. Los puntos Pe indican la precipitación efectiva diaria registrada durante el ciclo del cultivo y los puntos Riego Neto recogen la altura de lamina de agua $(\mathrm{mm} \circ \mathrm{l} / \mathrm{m} 2)$ aplicada con los diferentes riegos.

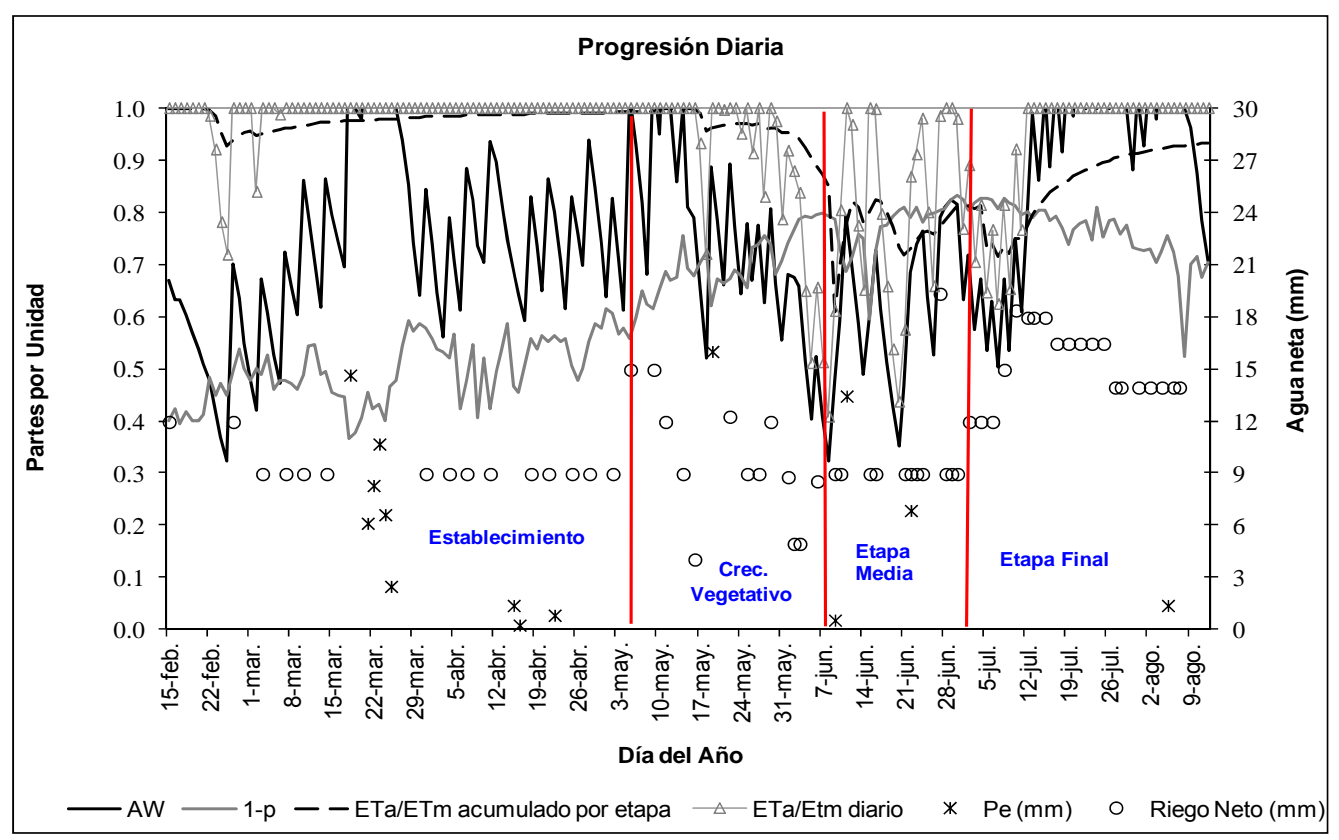

Figura 2. Evolución del contenido de agua aplicado para el cultivo de cebolla. Manejo real aplicado por agricultor (E1).

En este escenario, el riego bruto aportado se estimó en $7907 \mathrm{~m}^{3} /$ ha (estimado a partir horas de funcionamiento del sistema). La relación Evapotranspiración real /Evapotranspiración máxima del cultivo (ETa/ETm) del 90\%, indica que se realizó un manejo deficitario. Por etapas, las relaciones ETa/ETm fueron del $99 \%$ en la etapa de establecimiento, del $85 \%$ en la etapa de crecimiento vegetativo (estrés sufrido entre los días 21 de mayo hasta 6 de junio), del $81 \%$ en la etapa media (estrés observado en la Figura 2 entre los días 8 de junio hasta 03 de julio), y del 93\% en la etapa final (estrés moderado sufrido entre los días 4 hasta 12 de julio). La percolación estimada con MOPECO fue de 538 $\mathrm{m}^{3} / \mathrm{ha}$, de los que $226 \mathrm{~m}^{3} /$ ha se debieron a las lluvias ocurridas en el mes de marzo, (primera etapa del cultivo) y los $312 \mathrm{~m}^{3} /$ ha por el riego (Etapa Final). 
Las pautas del manejo del riego del agricultor fueron las siguientes: durante la etapa de establecimiento, riegos de $1 \mathrm{~h}(6 \mathrm{~mm}) 2$ veces a la semana. A partir de la etapa de crecimiento vegetativo hasta mitad de la etapa media, riegos de hasta $2 \mathrm{~h}(12 \mathrm{~mm})$, tres veces por semana. A partir de la mitad de la etapa media hasta el final del ciclo del cultivo, los riegos fueron de hasta $3 \mathrm{~h}$, con una frecuencia de 4 o 5 veces a la semana. El rendimiento final obtenido fue de $56.419 \mathrm{~kg} / \mathrm{ha}$ (Tabla 2), inferior a los $70.000 \mathrm{~kg} / \mathrm{ha}$ (rendimiento potencial considerado con base al obtenidos en otras parcelas en la misma zona en el año 2015). Este descenso del $24 \%$ respecto a producción máxima puede ser debido mayoritariamente al estrés de aproximadamente el $10 \%$ al que fue sometido el cultivo. En otras parcelas de seguimiento en la zona, para una relación ETa/ETm del 93\%, se obtuvieron rendimientos de $65682 \mathrm{~kg} / \mathrm{ha}$.

En el escenario E2, donde se utilizan las recomendaciones semanales de consumos del SIAR ${ }_{\mathrm{CM}}$, el aporte en forma de riego bruto estimado es de $8.724 \mathrm{~m}^{3} / \mathrm{ha}$, para una relación $\mathrm{ETa} / \mathrm{ETm}$ del $96 \%$. Por etapas, as relaciones $\mathrm{ETa} / \mathrm{ETm}$ fueron del $96 \%$ en la etapa de establecimiento, del $88 \%$ en la etapa de crecimiento vegetativo, $100 \%$ en la etapa media y $99 \%$ en la etapa final. Según los datos simulados por el programa MOPECO, la percolación estimada sería de $787 \mathrm{~m}^{3} / \mathrm{ha}$, de los que $323 \mathrm{~m}^{3} /$ ha se debieron a las lluvias ocurridas en el mes de marzo y los $464 \mathrm{~m}^{3} / \mathrm{ha}$ por el riego

Las pautas del manejo del riego basada en las recomendaciones semanales del SIAR $_{\mathrm{CM}}$ fueron: durante la etapa de establecimiento, riegos de hasta $3,5 \mathrm{~h}(21 \mathrm{~mm})$, una vez a la semana. En la etapa de crecimiento vegetativo, riegos de hasta $3 \mathrm{~h}(18 \mathrm{~mm})$ dos veces a la semana. En la etapa media, los riegos fueron de hasta $4 \mathrm{~h}(24 \mathrm{~mm})$, con una frecuencia de 2 o 3 veces a la semana y en la etapa final los riegos fueron de hasta $4 \mathrm{~h}(24 \mathrm{~mm}) 3$ veces a la semana. El rendimiento estimado fue de $67.150 \mathrm{~kg} / \mathrm{m}^{3}$ (Tabla 2), lo que representa un incremento de $19 \%$ con respecto a producción real obtenida.

En el escenario E3, el riego bruto aportado se estimó en $7907 \mathrm{~m}^{3} / \mathrm{ha}$ (aportando el mismo volumen de agua total que el agricultor (E1) variando la pauta de riegos) alcanzado una $\mathrm{ETa} / \mathrm{ETm}$ de $97 \%$, lo que conlleva un ligero estrés. Las relaciones ETa/ETm serían de $98 \%$ en la etapa de establecimiento, $92 \%$ en la etapa de crecimiento vegetativo, $97 \%$ en la etapa media y $99 \%$ en la etapa final. La percolación estimada con MOPECO fue de 158 $\mathrm{m}^{3} /$ ha, debido a las lluvias ocurridas en el mes de marzo (primera etapa del cultivo).

Las pautas del manejo del riego en este escenario fueron: durante la etapa de establecimiento riegos de hasta $1,5 \mathrm{~h}(9 \mathrm{~mm})$ dos veces a la semana (intervalo entre riegos de 4 días). Durante la mitad de la etapa de crecimiento vegetativo, riegos de hasta $2,5 \mathrm{~h}$ (15 $\mathrm{mm}) 2$ o 3 veces a la semana (intervalo de riegos de 3 días). A partir de la mitad de la segunda etapa (crecimiento vegetativo) hasta la primera semana de la etapa final, riegos de hasta $3 \mathrm{~h}$ (18 mm), con intervalo de 2 días (3 o 4 veces a la semana). En el resto de la etapa final riegos de hasta $2,1 \mathrm{~h}(13 \mathrm{~mm}) 3$ o 4 veces a la semana (intervalo de 2 días).

En la Figura 3 se representa la distribución del agua aplicada en las distintas etapas de desarrollo fenológico para los escenarios E1 y E3, donde el volumen total en el ciclo es el mismo. En la etapa de establecimiento y la etapa final el agricultor (E1) aplica un volumen superior al estimado en el E3, mientras que en la etapa de crecimiento vegetativo y etapa media el aporte fue inferior.

La principal diferencia entre ambos manejos, radica en el momento de aplicación del déficit, concentrado en el E3 durante la etapa de crecimiento vegetativo (ETa/ETm de 92\%) pues durante los periodos vegetativos y de maduración, el cultivo parece ser menos sensible al déficit de agua (Shock et al., 2000; Kadayifci et al., 2005; Bekele and Tilahun, 2007). En el E3, durante la etapa final, se reduce la aportación de agua, lo que limita la percolación (en E1 la percolación en esta etapa fue de $312 \mathrm{~m}^{3} / \mathrm{ha}$ ). Este manejo permite aumentar el aporte en la etapa de máxima sensibilidad al estrés (etapa media), aumentando el rendimiento en un $18,4 \%$ (66.801 kg/ha en el E3, frente a los $56.419 \mathrm{~kg} / \mathrm{ha}$ del E1). 


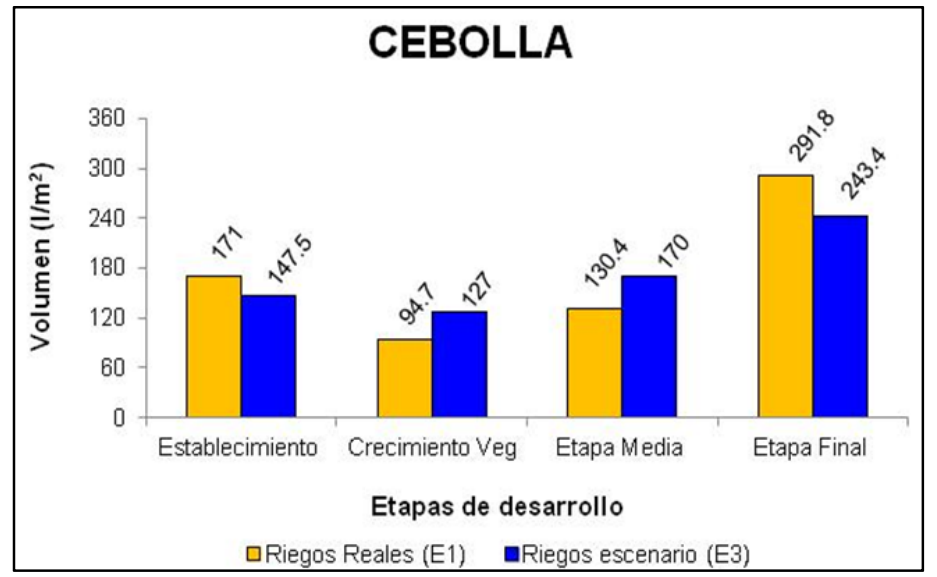

Figura 3: Distribución del agua aportada en las distintas etapas de cebolla para los los escenarios E1 y E3 (igual volumen total).

En el escenario 4, donde se simuló un manejo sin estrés, de máximo rendimiento, el programa estima un riego bruto de $8.256 \mathrm{~m}^{3} /$ ha con una ETa/ETm final y por etapas de $100 \%$. La percolación estimada con MOPECO fue de $138 \mathrm{~m}^{3} / \mathrm{ha}$, debido a las lluvias en el mes de marzo.

Las pautas del manejo del riego en este escenario fueron: durante la etapa de establecimiento riegos de hasta $1,5 \mathrm{~h}(9 \mathrm{~mm})$ aplicados con intervalo de riegos de 2 o 3 días. En la etapa de crecimiento vegetativo riegos de hasta $2,6 \mathrm{~h}(15,4 \mathrm{~mm}) 3$ veces a la semana. En la etapa media riegos de hasta $3,5 \mathrm{~h}(21,3 \mathrm{~mm}) 3$ veces a la semana y en la etapa final riegos de hasta $3,6 \mathrm{~h}(21,5 \mathrm{~mm}) 2$ a 4 veces a la semana. El rendimiento estimado fue de $69.769 \mathrm{~kg} / \mathrm{ha}$.

En la Figura 4 se representa los riegos semanales aplicados en el E1 y los propuestos en el resto de los distintos escenarios, así como la evolución del volumen acumulado por semanas. En la Figura 4a, el E1 frente al E2, durante la primera etapa los riegos aplicados por el agricultor fueron mayores que el recomendado en el E2. En la etapa de crecimiento vegetativo y la etapa media riega por debajo del E2 y en la etapa final los riegos, en su mayoría, fueron superiores al recomendado en E2.

La Figura 4b, se observa la comparación entre E1 y E3 que durante la etapa de establecimiento los riegos reales aplicados fueron superiores durante el período de 2 de marzo hasta 12 de abril. Durante la Etapa de crecimiento vegetativo y Etapa media el E3 recomienda regar más, sin embargo en la etapa final recomienda regar menos al aplicado en el E1.

La comparación entre E1 y E4 (Figura 4c), en la etapa de establecimiento el agricultor ha regado más que el propuesto en el período de 2 a 15 de marzo. Durante las etapas de crecimiento vegetativo y media hasta el día 12 de julio (inicio de la etapa final) ha regado por debajo del recomendado en E4. A partir de mediados de la etapa final ha regado por encima del indicado en E4.

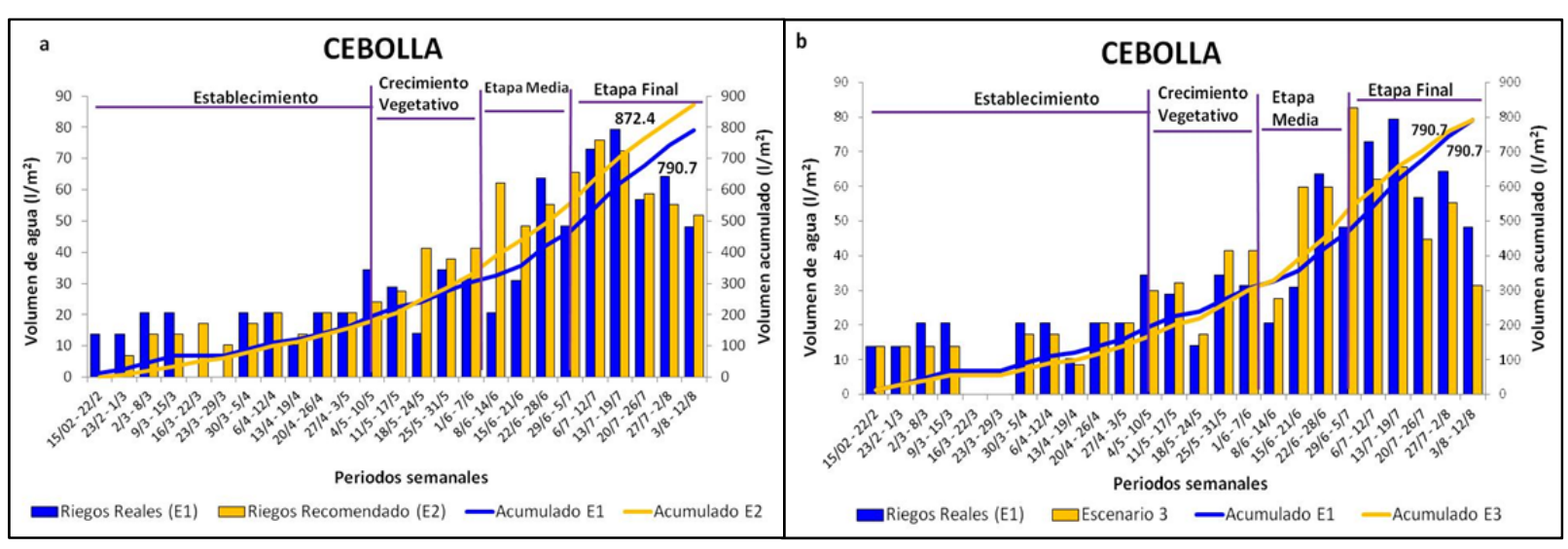




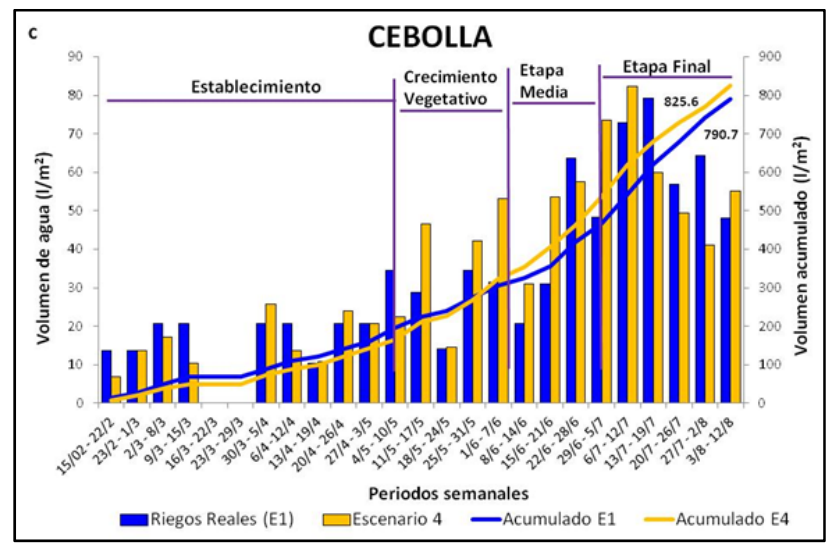

Figura 4. (a) Comparación entre los riegos semanales aplicados (E1) y los propuestos en la simulación del E2; (b) Comparación entre E1 y los propuestos en la simulación del E3; (c) Comparación entre E1 y los propuestos en la simulación del E4.

\section{2) Evaluación de los Costes}

En la Tabla 2 se presenta los ingresos y los costes para los cuatro escenarios: E1 (real), E2 (recomendación de consumos máximos semanales, SIAR $R_{C L M}$ ), E3 (mismo volumen del E1 aplicado con distinto intervalo y dosis de riego) y E4 (riego con máxima producción).

Tabla 2. Resumen de producción, Ingresos y los Costes para el cultivo de Cebolla en la parcela estudio.

\begin{tabular}{|c|c|c|c|c|}
\hline & E1 & E2 & E3 & E4 \\
\hline Producción (kg/ha) & 56419 & 67150 & 66801 & 69769 \\
\hline Ingresos + PAC (€/ha) & 8198,7 & 9701 & 9652,3 & 10067,8 \\
\hline Costes externos $(€ / \mathrm{ha})$ & 2489,8 & 2590,6 & 2489,8 & 2532,9 \\
\hline Costes calculados $(€ / \mathrm{ha})$ & 2134,6 & 2530,5 & 2134,5 & 2528,7 \\
\hline Costes estimados $(€ / \mathrm{ha})$ & 779,7 & 915 & 883 & 941,3 \\
\hline Coste Fijo $(€ / \mathrm{ha})$ & 130 & 130 & 130 & 130 \\
\hline Costes Totales $(€ / \mathrm{ha})$ & 5534,1 & 6166,2 & 5637,9 & 6132,9 \\
\hline
\end{tabular}

Para calcular los costes de los escenarios se utilizaron los mismos datos de materia prima y laboreos facilitados por el agricultor, y por lo tanto, la diferencia entre los costes es la cantidad de volumen bruto aportado en cada escenario y el incremento de los costes de mano de obra derivado del tiempo de funcionamiento del sistema de riego. El precio de venta informado por el agricultor fue de $0,14 € / \mathrm{kg}$. Se puede observar que los distintos manejos influyeron en la producción, donde E4 presenta mayor producción estimada $(69.769 \mathrm{~kg} / \mathrm{ha}), 24 \%$ más que la producción real y consecuentemente mayores ingresos, debido a la mayor producción estimada. El E2 presenta mayores costes por estimar un aporte mayor de volumen de riego bruto. Es importante destacar que en las simulaciones para E1 y E3, utiliza la misma cantidad de agua, sin embargo una mejor programación del manejo conlleva a un incremento en la producción de $18,4 \%$ en E3 $(66.801 \mathrm{~kg} / \mathrm{ha})$ respecto a producción real. El E2 presenta una producción estimada en $67150 \mathrm{~kg} / \mathrm{ha}$, lo que supone un incremento de $19 \%$ respecto a real obtenida.

\section{3) Indicadores}

La Tabla 3 presenta los valores de los indicadores de los cuatro escenarios estudiados. En los E1 y E3 se da el menor valor de SRA. En el escenario 1, el cultivo sufrió un estrés centrado en la Etapa de crecimiento vegetativo (ETa/ETm de $85 \%)$ y en la Etapa media (ETa/ETm de $81 \%)$. En E3 el cultivo presenta estrés en la Etapa de crecimiento 
vegetativo (ETa/ETm de 92\%). En estos dos escenarios los valores de SRA son iguales al usar la misma cantidad de riego bruto aportado, pero con distribución distinta. A pesar del estrés mas fuerte, el manejo aplicado por el agricultor se clasifica a nivel global como bien regado, al igual que en el resto de los escenarios. Esa clasificación indica que las necesidades de los cultivos se ven satisfechas correctamente. El efecto del estrés sometido al cultivo en los escenarios E1 y E3 se puede observar más claramente al analizar el indicador IP, que presenta menor valor $(0,81)$ en el E1. EI menor valor de IP en E1 puede ser asignado al estrés sufrido en la tercera etapa, ya que esta es la etapa de mayor sensibilidad al estrés en el cultivo de la cebolla. En ésta la falta de agua puede repercutir más negativamente en el rendimiento final del cultivo. En el E2, ofrece un índice de producción alto $(0,96)$ al igual que el E3 y el E4 (IP de 0,95 y 1,00 respectivamente).

Tabla 3. Indicadores evaluados para los distintos escenarios en el cultivo de cebolla

\begin{tabular}{|l|c|c|c|c|}
\hline Indicador & E 1 & E 2 & E 3 & E 4 \\
\hline SRA (\%) & 0,98 & 1,07 & 0,98 & 1,01 \\
\hline IP & 0,81 & 0,96 & 0,95 & 1 \\
\hline MB $(€ / \mathrm{ha})$ & 2664,6 & 3534,8 & 4014,4 & 3934,8 \\
\hline PAA $\left(\mathrm{kg} / \mathrm{m}^{3}\right)$ & 7,14 & 7,70 & 8,45 & 8,45 \\
\hline
\end{tabular}

SRA = Suministro relativo de agua; IP, índice de producción; MB, margen bruto; PAA, productividad agronómica del agua.

Analizando el indicador Margen Bruto, (Tabla 3) se puede comprobar que el manejo aplicado por el agricultor derivó en un menor valor si se compara con el resto. En el E1 (riego bruto estimado en $7.907 \mathrm{~m}^{3} / \mathrm{ha}$ ) y E2, basada en las recomendaciones semanales del $\mathrm{SIAR}_{\mathrm{CM}}$, la programación recomienda aplicar un mayor valor de riego bruto $\left(8.724 \mathrm{~m}^{3} / \mathrm{ha}\right)$, que conlleva un aumento de los costes en $11,4 \%$, sin embargo aumenta la producción estimada en $67150 \mathrm{~kg} / \mathrm{ha}$ (19\% de incremento), y el MB en un $32 \%$.

Comparando el E1 con el E3, con aporte de riego bruto estimado en $7.907 \mathrm{~m}^{3} / \mathrm{ha}$, el coste total en el E3 es ligeramente mayor debido al seguro agrario, considerado en la metodología, como $1 \%$ del valor de la producción final. En eses escenarios se puede observar claramente el efecto del manejo aplicado, utilizando la misma cantidad de riego bruto de forma distinta, puede proporcionar aumento de la producción del 18,4\% (66.801 kg/ha), que repercute en un aumento del $50 \%$ del MB en el E3 (Tabla 3).

En el manejo propuesto en $\mathrm{E} 4$, con máxima producción, el riego bruto recomendado es de $8.256 \mathrm{~m}^{3} /$ ha, un $4,4 \%$ más que en el E1 $\left(7.907 \mathrm{~m}^{3} /\right.$ ha) que repercute en un incremento de los costes del $11 \%$, un aumento de producción del $24 \%$, que supone un $47,6 \%$ más en el MB (Tabla 3).

El indicador PAA se ha utilizado para analizar la diferencia entre el uso o no de las recomendaciones del SIAR $R_{C M}$. El E1 presenta una PAA de $7,14 \mathrm{~kg} / \mathrm{m}^{3}$, mientras que en el E3 y E4, se estima la mayor PAA, que asciende a $8,45 \mathrm{~kg} / \mathrm{m}^{3}$ en los dos escenarios. Para E2 el valor estimado sería menor que en el E3 y $\mathrm{E} 4$, con $7,70 \mathrm{~kg} / \mathrm{m}^{3}$. En un estudio similar, Montoro et. al (2008), estima un valor superior, con una media de PAA en la cebolla de $10,13 \mathrm{~kg} / \mathrm{m}^{3}$ para una producción media de $65.125 \mathrm{~kg} / \mathrm{ha}$ y $6.472 \mathrm{~m}^{3} / \mathrm{ha}$ de agua aplicada (media de los años de 2000 hasta 2005). Las condiciones climáticas del año 2015 con temperaturas más altas de los valores medios, pudo repercutir en los resultados, al requerir el cultivo un mayor volumen de agua para satisfacer las necesidades hídricas. Con los datos reales y los escenarios simulados se puede observar el efecto de distintos manejos en la producción, en los costes, el MB y a utilización de indicadores que posibilitan evaluar el posible beneficio de una correcta programación de riegos.

\section{4) Conclusiones}


La consideración de las recomendaciones semanales de carácter general de consumos máximos ofrecidas por el SIAR $\mathrm{CM}_{\mathrm{CM}}$, frente a un manejo del riego basado en la experiencia, repercute en un aumento del rendimiento del cultivo de cebolla cuya cuantificación dependerá del manejo que esté realizando. En el caso del estudio el aumento del rendimiento ha sido del $19 \%$, con una mejora en la eficiencia del uso de agua del $8 \%$ y del Margen Bruto estimado en $32 \%$. Una programación de riegos, basada en un balance de agua diario del suelo, permite ajustar el agua aplicada frente a la demandada por el cultivo, aproximando los rendimientos reales a los potenciales, limitando el agua perdida por percolación. En este caso el aumento del rendimiento ha sido del $18,4 \%$, con una mejora en la eficiencia del uso de agua del $18 \%$ y del Margen Bruto estimado en $50 \%$.

En una gran zona regable, la información general que proporcionan los SARs o la correcta programación de riegos basada en un balance de agua en el suelo a nivel de parcela mejora los rendimientos, el margen bruto y la eficiencia de los cultivos en distintos niveles.

\section{5) Agradecimientos}

Este trabajo fue realizado con el apoyo de CAPES, Coordinación de Perfeccionamiento de Personal de Nivel Superior - Brasil.

\section{6) Bibliografía}

Allen, R.G., Pereira, L.S., Raes, D., Smith, M., Crop evapotranspiration: guidelines for computing crop water requirements. Irrigation and Drainage. Paper 56. FAO, Rome, Italy, 1998.

Bekele, S., Tilahun, K., 2007. Regulated déficit irrigation scheduling of onion in a semiarid region of Ethiopia. Agricultural Water Management 89, 148-152.

Bos, M.G.; Murray-Rust, D.H.; Merrey, D.J.; Johnson, H. G. y Snellen, W.B. 1994. Methodologies for assessing performances of irrigation and drainage management. Irrigation and Drainage Systems, 7:231-261.

de Juan, J.A., Ortega, J.F., Tarjuelo, J.M., 2003. Sistemas de cultivo. Evaluación de itinerarios técnicos. Ediciones Mundi-Prensa, Madrid, Spain.

Domínguez A., Jiménez. M., Tarjuelo J.M., de Juan. J. A., Martínez-Romero A., Leite. K. N., 2012. Simulation of onion crop behavior under optimized regulated deficit irrigation using MOPECO model in a semi-arid enviroment. Agricultural Water Management. 113, 64-75 Q1.

FAO (Food and Agricultura Organization of the United Nations), 2003. Unlocking the Water Potencial of Agriculture. Roma, Italia.

Kadayifci, A., Tuylu, G.I., Ucar, Y., Cakmak, B., 2005. Crop water use of onion (Allium cepa L.) in Turkey. Agricultural Water Management 72, 59-68.

Levine G, 1982. Relative Water Supply: An explanatory variable for irrigation systems. Technichal Report No. 6. Cornell University. Ithaca, New York. EEUU.

Martín de Santa Olalla, F.J., Domínguez-Padilla, A., Lopez, R., 2004. Production and quality of the onion crop (Allium cepa L.) cultivated under controlled deficit irrigation conditions in a semi-arid climate. Agricultural Water Management 68, 77-89.

Martínez-Valderrama, J., Ibáñez, J., Alcalá, F.J., Domínguez, A., Yassin, M., Puigdefábregas, J., 2011. The use of a hydrological-economic model to assess sustainability in groundwater-dependent agriculture in drylands. Journal of Hydrology 402, 80-91.

NRCS, 2004. Part 630 Hydrology. National Engineering Handbook. Chapter 10. Estimation of Direct Runoff from Storm Rainfall. Natural Resources Conservation Service. United States Department of Agriculture.

SCS, 1972. Section 4: Hydrology. National Engineering Handbook. Soil Conservation Service, United States Department of Agriculture.

Shock,C. C. Feibert, E. B. G., Saunders, L. D. , 2000. Irrigation criteria for drip-irrigated onions. HortScience 35, 63-66.

Stewart, J. I., Hagan, R. M., Pruitt, W. O., Kanks, R. J., Riley, J. P., Danilson, R. E., Franklin, W. T., Jackson, E. B., 1977. Optimizing Crop Production Trough Control of Water and Salinity levels. Utah Water Res. Lab. PWRG 151-1, UT, USA. 